\title{
CONTROL/FAULT DIAGNOSIS INTERACTIONS ANALYSIS FOR STRUCTURED BILINEAR SYSTEMS
}

\author{
S. Canitrot , T. Boukhobza , F. Hamelin and \\ S. Martinez-Martinez
}

\author{
Research Centre in Automatic Control (CRAN - CNRS UMR \\ 7039) Nancy University, BP 239, 54506 Vandouvre Cedex \\ France Phone: 33383684464 Fax: 33383684462 \\ email: sebastien.canitrot@cran.uhp-nancy.fr
}

\begin{abstract}
This work is dedicated to the study of control/fault diagnosis interactions for structured bilinear systems using a graph-theoretic approach. According to the fact that constant control inputs make possible the representation of a bilinear system by a linear one, some propositions provide the sufficient conditions for active-BFPRG (Bilinear Fundamental Problem of Residual Generation) in this particular case. These conditions are easy to check because they are based on the comparison of expressions and on finding paths in a digraph.
\end{abstract}

Keywords: Structural properties, bilinear systems, fault diagnosis, graph theory.

\section{INTRODUCTION}

Fault detection and isolation (FDI) problem has received considerable attention in the two last decades (Chen and Patton, 1999; Frank, 1996; Gertler, 1998). Detection and isolation of incipient faults is important for safety critical systems where a malfunction can cause human and material damages.

Bilinear systems (BLS) are amongst the simplest nonlinear systems and therefore are particularly adapted to analysis compared to more complicated nonlinear systems. They can be used to represent a wide range of physical, chemical, biological and social systems, as well as manufacturing processes which cannot be satisfactorily controlled under the linearity assumption. The properties and behavior of bilinear systems have been investigated and a number of useful results have been derived.

Up to now, the problem of residual generation for BLS is mainly treated using algebraic or geometric tools (Yang and Saif, 1995; Hammouri et al., 2001). One of the most important results about FDI of BLS is given in (Hammouri et al., 2001). Using a geometric approach, the authors provide necessary and sufficient conditions for the solvability of the so-called Bilinear Fundamental Problem of Residual Generation (BFPRG). This problem extends the Fundamental Problem of Residual Generation (FPRG) defined and solved for linear systems in (Massoumnia et al., 1989). The solvability conditions of such a problem for nonlinear systems, which are affine in the control inputs and in the failure modes, have been also given in (Hammouri et al., 1999; De Persis and Isidori, 2001).

Many studies on structured systems are related to the graph-theoretic approach. Until now, this approach is mainly dedicated to linear systems. (Dion et al., 2003) reviews the most significant results in this area. From these works, it results that the graph-theoretic approach provides simple and elegant solutions and so is very well suited to analyze large scale or/and uncertain systems. The quality of the results obtained for linear systems by using a graphic approach has motivated us to apply the latter to the problem of residual generation for structured bilinear systems (sBLs).

The main contribution of this note is the study of the so-called "active"-BFPRG. This problem, as we define it more precisely later, is related to the existence of 
particular control inputs which make the BFPRG solvable. Indeed, reducing the FDI problem to the usual BFPRG is quite restrictive, since the control input can be used to achieve the detection and the isolation of faults. Effectively, in practice, many FDI strategies use inputs, or more generally, some specific functioning modes to detect and to isolate the faults occurring on the system.

In this context, it seems interesting to study the input influence on the solvability of the BFPRG. More precisely, we study two kinds of control inputs. Firstly, we search to solve the active-BFPRG considering all control inputs as generic constant. Next, we set to zero some inputs considering the others constant.

All the results provided in this paper are based on the graph-theoretic approach. The solvability conditions for residual generation problem are related to comparison of expressions and to computation of paths in a digraph. This makes our approach very well suited to study large scale and/or uncertain systems.

The paper is organized as follows: after section 2, which is devoted to the active-BFPRG formulation, a digraph representation of structured bilinear systems is defined in section 3. Some sufficient conditions for the solvability of active-BFPR, are enounced in section 4. Finally, some concluding remarks are made.

\section{PROBLEM STATEMENT}

We consider sBLS of the form:

$\Sigma_{\Lambda}:\left\{\begin{array}{l}\dot{x}(t)=A_{0} x(t)+\sum_{\ell=1}^{m} u_{\ell}(t) A_{\ell} x(t)+E f_{1}(t)+H w(t) \\ y(t)=C x(t)\end{array}\right.$

where $x(t) \in \mathbb{R}^{n}, u(t) \in \mathbb{R}^{m}, f_{1}(t) \in \mathbb{R}, w(t) \in \mathbb{R}^{d}$ and $y(t) \in \mathbb{R}^{p}$ are respectively the state, the input, the fault, the disturbance and the output vectors. In this study, we treat the case of system with only one fault $f_{1}$. However, if the system is corrupted by several faults, the study can also be made to each fault considering other faults as disturbances.

$A_{\ell}$, for $\ell=0, \ldots, m, C, E$ and $H$ represent matrices of appropriate dimensions, which elements are either fixed to zero or assumed free nonzero parameters. We can parameterize these nonzero entries by real (nonzero) parameters $\lambda_{i}, i=1, \ldots, h$ forming a parameter vector $\Lambda=\left(\lambda_{1}, \ldots, \lambda_{h}\right)^{T} \in \mathbb{R}^{h}$. If all the nonzeros parameters $\lambda_{i}$ are fixed, we obtain an admissible realization of structured system $\Sigma_{\Lambda}$. Theoretic properties of each realization can be studied according to the values of $\lambda_{i}$. We say that a property is true generically if it is true for almost all the realizations of structured system $\Sigma_{\Lambda}$. Here, "for almost all the realizations" is to be understood as "for all parameter values $\left(\Lambda \in \mathbb{R}^{h}\right)$ except for those in some proper algebraic variety in the parameter space". The proper algebraic variety for which the property is not true is the zero set of some nontrivial polynomial with real coefficients in the system parameters $\lambda_{1}, \lambda_{2}, \ldots, \lambda_{h}$ or equivalently it is an algebraic variety which has Lebesgue measure zero.

Usually, the fault diagnosis problem and the control problem are separated but there exist some control/diagnosis interactions studies as those concerning FTC (Fault Tolerant Control). In this note we make a complementary study of these interactions. Our aim is to provide sufficient conditions for the generic solvability of the following problem.

Consider sBLs $\Sigma_{\Lambda}$, we define the active-BFPRG as the problem of finding filter of the form:

$$
\left\{\begin{array}{l}
\dot{z}(t)=\phi(z(t), y(t))+\varphi(z(t), y(t)) u(t) \\
\delta(t)=h(z(t), y(t))
\end{array}\right.
$$

with $z(t) \in \mathbb{R}^{r}$ and $\delta(t) \in \mathbb{R}$, such that it generically exists an input $u(t)$ so that

- if $f_{1}(t)=0$ then $\delta(t) \rightarrow 0$ for all initial conditions.

- else, $\delta(t) \neq 0$ for all initial conditions

That is to say, $\delta(t)$ is insensitive to $w(t)$.

The "classical" BFPRG is to find a filter (2) such that $\delta(t)$ enables the detection of $f_{1}(t)$ for any admissible inputs $u(t)$. It is well-known that in bilinear systems, the fault detection is affected by the control input. In fact, a specific control value can make possible the detection of a fault, which is not detectable for all other values of $u(t)$. In another way, a specific control value can make impossible the detection of a fault, which is detectable for all other values of $u(t)$. Like many structural properties, we think that the interactions between control and fault diagnosis can be more easily handled using a graph-theoretic approach defined in the next section.

\section{GRAPHICAL REPRESENTATION OF STRUCTURED BILINEAR SYSTEMS}

The graph, directly obtained from the system structure, is interesting for several reasons. In particular, the graph-based results, given by weak burden calculation, are intuitive and are easily applicable to large scale systems.

\subsection{Digraph definition for SBLS}

The digraph associated to bilinear system $\Sigma_{\Lambda}$ is noted $\mathcal{G}\left(\Sigma_{\Lambda}\right)$ and is constituted by a vertex set $\mathcal{V}$ and an edge set $\mathcal{E}: \mathcal{G}\left(\Sigma_{\Lambda}\right)=(\mathcal{V}, \mathcal{E})$. The vertices are associated to the variables of $\Sigma_{\Lambda}$ and the directed edges represent links between these variables. To differentiate variables from their corresponding vertices, the latter are written in bold fonts.

The vertex set is $\mathcal{V}=\mathbf{X} \cup \mathbf{Y} \cup \mathbf{W} \cup\left\{\mathbf{f}_{\mathbf{1}}\right\}$ where $\mathbf{X}=$ $\left\{\mathbf{x}_{1}, \ldots, \mathbf{x}_{\mathbf{n}}\right\}$ is the set of state vertices, $\mathbf{Y}=\left\{\mathbf{y}_{\mathbf{1}}, \ldots, \mathbf{y}_{\mathbf{p}}\right\}$ is the set of output vertices, $\mathbf{W}=\left\{\mathbf{w}_{\mathbf{1}}, \ldots, \mathbf{w}_{\mathbf{d}}\right\}$ is the 
set of disturbance vertices and $\mathbf{f}_{\mathbf{1}}$ is the fault vertex. The edge set is $\mathcal{E}=\bigcup_{\ell=0}^{m} A_{\ell}$-edges $\cup C$-edges $\cup E$-edges $\cup$ $H$-edges where $A_{\ell}$-edges $=\left\{\left(\mathbf{x}_{\mathbf{i}}, \mathbf{x}_{\mathbf{j}}\right) \mid A_{\ell}(j, i) \neq 0\right\}$ for $\ell=0, \ldots, m, C$-edges $=\left\{\left(\mathbf{x}_{\mathbf{i}}, \mathbf{y}_{\mathbf{j}}\right) \mid C(j, i) \neq 0\right\}$, $E$-edges $=\left\{\left(\mathbf{f}_{\mathbf{1}}, \mathbf{x}_{\mathbf{j}}\right) \mid E(j) \neq 0\right\}$ and $H$-edges $=$ $\left\{\left(\mathbf{w}_{\mathbf{i}}, \mathbf{x}_{\mathbf{j}}\right) \mid H(j, i) \neq 0\right\} .\left(\mathbf{v}_{\mathbf{i}}, \mathbf{v}_{\mathbf{j}}\right)$ denotes a directed edge from begin vertex $\mathbf{v}_{\mathbf{i}} \in \mathcal{V}$ to end vertex $\mathbf{v}_{\mathbf{j}} \in \mathcal{V}$.

We take the following notation : $\bar{A}_{0}$-edges = $A_{0}$-edges $\cup C$-edges $\cup E$-edges $\cup H$-edges and for $\ell=1, \ldots, m, \bar{A}_{\ell}$-edges $=A_{\ell}$-edges. To each edge $e \in \bar{A}_{\ell}$-edges is associated an unique indice $\mathrm{u}_{\ell}$ and we indicate $u_{\ell}$ over each edge $e$ in the digraph representation. If an edge $e_{1} \in \bar{A}_{\ell_{1}}$-edges and an edge $e_{2} \in \bar{A}_{\ell_{2}}$-edges have the same begin and end vertices, only one edge is represented and the information over the edge will be $u_{\ell_{1}}, u_{\ell_{2}}$ with $\ell_{1}<\ell_{2}$.

Example 1. Consider sBLs defined by:

$A_{0}=\left(\begin{array}{ccccc}0 & 0 & 0 & 0 & 0 \\ 0 & 0 & 0 & 0 & 0 \\ 0 & 0 & 0 & \lambda_{1} & 0 \\ \lambda_{2} & \lambda_{3} & 0 & 0 & 0 \\ 0 & 0 & \lambda_{4} & 0 & 0\end{array}\right), A_{1}=\left(\begin{array}{ccccc}0 & 0 & 0 & 0 & 0 \\ 0 & 0 & 0 & 0 & 0 \\ 0 & 0 & 0 & 0 & 0 \\ 0 & \lambda_{5} & 0 & 0 & 0 \\ 0 & 0 & 0 & \lambda_{6} & 0\end{array}\right), A_{2}=$

$\left(\begin{array}{ccccc}0 & 0 & 0 & 0 & 0 \\ 0 & 0 & 0 & 0 & 0 \\ \lambda_{7} & 0 & 0 & 0 & 0 \\ 0 & 0 & 0 & 0 & 0 \\ 0 & 0 & 0 & 0 & 0\end{array}\right), E=\left(\begin{array}{c}0 \\ \lambda_{8} \\ 0 \\ 0 \\ 0\end{array}\right), H=\left(\begin{array}{c}\lambda_{9} \\ 0 \\ 0 \\ 0 \\ 0\end{array}\right)$ and $C=\left(\begin{array}{lllll}0 & 0 & 0 & 0 & \lambda_{10}\end{array}\right)$.

This model is associated to digraph 1 .

We can see that the graph is easily obtained from the system structure.

\subsection{Notations and definitions}

- $P=\mathbf{v}_{\mathbf{r}_{\mathbf{0}}} \stackrel{u_{i_{1}}}{\longrightarrow} \mathbf{v}_{\mathbf{r}_{1}} \ldots \mathbf{v}_{\mathbf{r}_{\mathbf{k}-1}} \stackrel{u_{i_{k}}}{\longrightarrow} \mathbf{v}_{\mathbf{r}_{\mathbf{k}}}$ denotes $\mathbf{a}$ path $P$, which contains vertices $\mathbf{v}_{\mathbf{r}_{0}}, \ldots, \mathbf{v}_{\mathbf{r}_{\mathbf{k}}}$ and where $\left(\mathbf{v}_{\mathbf{r}_{\mathbf{j}}}, \mathbf{v}_{\mathbf{r}_{\mathbf{j}+1}}\right) \in \bar{A}_{i_{j+1}}$-edges for $j=0, \ldots, k-1 . \mathbf{v}_{\mathbf{r}_{0}}$ and $\mathbf{v}_{\mathbf{r}_{\mathbf{k}}}$ are respectively the begin vertex and the end vertex of $P$. We associate to $P$ an unique indice noted $\sigma(P)$ and defined by the ordered monomial $u_{i_{1}} \cdot u_{i_{2}} \cdot \ldots \cdot u_{i_{k}}$. A path $P$ is uniquely characterized by its indice and the vertices it covers. $P$ is an $Y$-topped path if its end vertex $\mathbf{v}_{\mathbf{r}_{\mathbf{k}}} \in \mathbf{Y}$.

- Some paths are disjoint if they have no common vertices.

- A set of $k_{l}$ disjoint paths from $\mathbf{V}_{\mathbf{0}} \in \mathcal{V}$ to $\mathbf{V}_{\mathbf{1}} \in \mathcal{V}$ is called a linking of dimension $k_{l}$ from $\mathbf{V}_{\mathbf{0}}$ to $\mathbf{V}_{\mathbf{1}}$. A linking $i$ composed of paths $P_{i_{1}}, P_{i_{2}}, \ldots, P_{i_{k}}$ is denoted

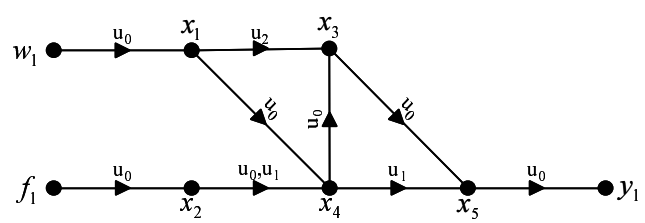

Figure 1. Digraph 1 associated to Example 1 $\mathrm{m}_{i}=\bigcup_{j=1}^{k} P_{i_{j}}$. A linking from $\mathbf{V}_{\mathbf{0}}$ to $\mathbf{V}_{\mathbf{1}}$ is maximum when $k_{l}$ is maximum. We denote by $\rho\left(\mathbf{V}_{\mathbf{0}}, \mathbf{V}_{\mathbf{1}}\right)$ the maximum number of disjoint paths from $\mathbf{V}_{\mathbf{0}}$ to $\mathbf{V}_{\mathbf{1}}$.

In example $1, \rho\left(\mathbf{W} \cup\left\{\mathbf{f}_{\mathbf{1}}\right\}, \mathbf{Y}\right)=1$.

- The set of all maximum linkings $\mathfrak{m}_{j_{1}}, \mathfrak{m}_{j_{2}}, \ldots, \mathfrak{m}_{j_{q}}$ from $\mathbf{V}_{\mathbf{0}}$ to $\mathbf{V}_{\mathbf{1}}$ is denoted by $\mathcal{M}\left(\mathbf{V}_{\mathbf{0}}, \mathbf{V}_{\mathbf{1}}\right)=\bigcup_{\ell=1}^{q} \mathrm{~m}_{j_{\ell}}$.

\section{ACTIVE-BFPRG}

The active-BFPRG is a quite vast problem because of the influence of the control input on the solution. Practically, it is tedious to solve this problem whatever the control, this is why in this paper we focus only on the simplest cases.

\subsection{Case of constant input}

In a first time, we consider that each control input is defined constant $(u(t)=u)$, this restriction can be justified by the fact that control inputs are constant in the steady state. In this case, structured bilinear system $\Sigma_{\Lambda}$ is equivalent to structured linear system $\Sigma_{\Lambda}^{1}$ with system parameters depending on the control value.

$$
\Sigma_{\Lambda}^{1}:\left\{\begin{array}{l}
\dot{x}(t)=\tilde{A}_{0} x(t)+E f_{1}(t)+H w(t) \\
y(t)=C x(t)
\end{array}\right.
$$

where $\tilde{A}_{0}=A_{0}+\sum_{\ell=1}^{m} u_{\ell} A_{\ell}$.

The BFPRG solvability can then be studied using the geometrical conditions given by (Hammouri et al., 2001). This approach requires the determination of $\mathcal{R}^{*}$, the minimal $(C, \mathcal{A})$-invariant subspace containing $H$, and $\mathcal{T}^{*}$, the minimal $(C, \mathcal{A})$-unobservability subspace containing $H$. The problem is solvable if $E \notin \mathcal{T}^{*}$. This study does not take part of the control input influence on fault detection as shown by example 2 .

Example 2. Consider sBLs defined by :

$$
\begin{aligned}
& A_{0}=\left(\begin{array}{cccc}
0 & 0 & 0 & 0 \\
0 & 0 & 0 & 0 \\
\lambda_{1} & 0 & 0 & 0 \\
0 & \lambda_{2} & 0 & 0
\end{array}\right), A_{1}=\left(\begin{array}{cccc}
0 & 0 & 0 & 0 \\
0 & 0 & 0 & 0 \\
0 & \lambda_{3} & 0 & 0 \\
0 & 0 & 0 & 0
\end{array}\right), H=\left(\begin{array}{c}
0 \\
\lambda_{4} \\
0 \\
0
\end{array}\right), E=\left(\begin{array}{c}
\lambda_{5} \\
0 \\
0 \\
0
\end{array}\right) \\
& \text { and } C=\left(\begin{array}{cccc}
0 & 0 & \lambda_{6} & 0 \\
0 & 0 & 0 & \lambda_{7}
\end{array}\right) \text {. }
\end{aligned}
$$

This system is represented by digraph 2 .

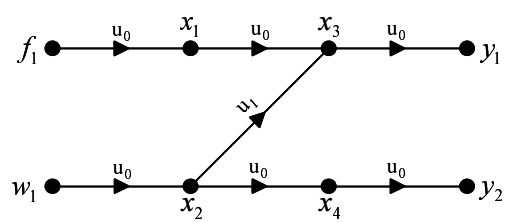

Figure 2. Digraph 2 associated to Example 2 
For this example, it can be shown that $\mathcal{R}^{*}=\operatorname{span}\left\{\left(\begin{array}{c}0 \\ \lambda_{4} \\ 0 \\ 0\end{array}\right),\left(\begin{array}{c}0 \\ 0 \\ \lambda_{3} \lambda_{4} \\ 0\end{array}\right),\left(\begin{array}{c}0 \\ 0 \\ 0 \\ \lambda_{2} \lambda_{4}\end{array}\right)\right\}$ and $\mathcal{T}^{*}=\mathbb{R}^{4}$.

Then, since $E \in \mathcal{T}^{*}$, the BFPRG has no solution.

If we consider that the control input is generically constant $\left(u_{1}(t)=u_{1}\right)$, the system matrices become:

$\tilde{A}_{0}=\left(\begin{array}{cccc}0 & 0 & 0 & 0 \\ 0 & 0 & 0 & 0 \\ \lambda_{1} & \lambda_{3} u_{1} & 0 & 0 \\ 0 & \lambda_{2} & 0 & 0\end{array}\right), H=\left(\begin{array}{c}0 \\ \lambda_{4} \\ 0 \\ 0\end{array}\right), E=\left(\begin{array}{c}\lambda_{5} \\ 0 \\ 0 \\ 0\end{array}\right)$ and $C=$ $\left(\begin{array}{cccc}0 & 0 & \lambda_{6} & 0 \\ 0 & 0 & 0 & \lambda_{7}\end{array}\right)$

In this case, the BFPRG is solvable using the geometrical conditions (Hammouri et al., 2001). Indeed, $\mathcal{R}^{*}=$ $\operatorname{span}\left\{\left(\begin{array}{c}0 \\ \lambda_{4} \\ 0 \\ 0\end{array}\right),\left(\begin{array}{c}0 \\ 0 \\ \lambda_{3} \lambda_{4} u_{1} \\ \lambda_{2} \lambda_{4}\end{array}\right)\right\}$ and $\mathcal{T}^{*}=\operatorname{span}\left\{\left(\begin{array}{l}0 \\ 1 \\ 0 \\ 0\end{array}\right),\left(\begin{array}{c}0 \\ 0 \\ \lambda_{3} \lambda_{4} u_{1} \\ \lambda_{2} \lambda_{4}\end{array}\right)\right\}$.

Since $E \notin \mathcal{T}^{*}$, the BFPRG is solvable.

In a practical point of view, the following filter makes possible the detection of fault $f_{1}$ :

$$
\left\{\begin{array}{l}
\dot{\hat{x}}(t)=A x(t)+K(y(t)-C \hat{x}(t)) \\
\delta(t)=Q(y(t)-C \hat{x}(t))
\end{array}\right.
$$

with matrices $K$ and $Q$ chosen such that $Q C(s I-$ $A+K C)^{-1} H=0$ and $Q C(s I-A+K C)^{-1} E \neq 0$. For the example, we have $K=\left(\begin{array}{ll}k_{11} & k_{12} \\ k_{21} & k_{22} \\ k_{31} & k_{32} \\ k_{41} & k_{42}\end{array}\right)$ and $Q=$ $\left(\begin{array}{ll}q_{1} & q_{2}\end{array}\right)$ under the constraints $q_{2}=-\frac{q_{1} \lambda_{3} u_{1} \lambda_{6}}{\lambda_{2} \lambda_{7}}, k_{11}=$ $-\frac{k_{12} \lambda_{2} \lambda_{7}}{\lambda_{3} u_{1} \lambda_{6}}$ and $k_{32}=\frac{\lambda_{3}\left(k_{42} \lambda_{2} \lambda_{7}-k_{31} \lambda_{2} \lambda_{6}+k_{41} \lambda_{3} \lambda_{6}\right)}{\lambda_{2}^{2} \lambda_{7}}$ (subject to stability constraints).

Consequently, it appears for this example that the active-BFPRG has a solution whereas the "classical" BFPRG is not solvable. The following proposition which is based on the results of (Commault et al., 2002) gives a sufficient condition to the active-BFPRG solvability. This condition is necessary and sufficient in the case of control inputs as generic constants.

\section{Proposition 1 :}

Consider sBLs $\Sigma_{\Lambda}$. In the case where all the control input components are constant, sBLs $\Sigma_{\Lambda}$ is equivalent to structured linear system $\Sigma_{\Lambda}^{1}$. The active-BFPRG is then solvable if:

$$
\rho\left(\mathbf{W} \cup\left\{\mathbf{f}_{\mathbf{1}}\right\}, \mathbf{Y}\right)=\rho(\mathbf{W}, \mathbf{Y})+1
$$

If the condition of proposition 1 is not satisfied then there are two possibilities for detecting fault $f_{1}$. The first one consists in increasing the value of $\rho(\mathbf{W} \cup$ $\left\{\mathbf{f}_{\mathbf{1}}\right\}, \mathbf{Y}$ ) (by addition of particular sensors to the system) and the second one consists in decreasing the value of $\rho(\mathbf{W}, \mathbf{Y})$ (using the influence of the control). We are interested in the second case.

The parameters of the obtained linear system are dependent of the control. In fact, setting some control components to zero results in edges removal in the

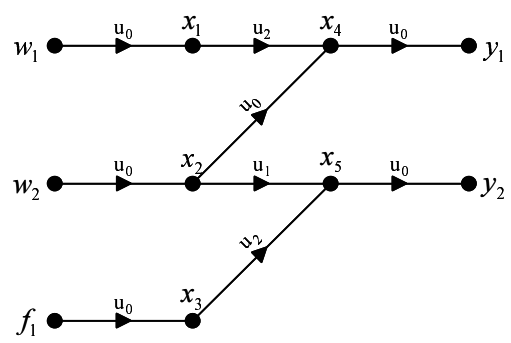

Figure 3. Digraph 3 associated to Example 3

digraph representation and then, the fault detection may be possible.

Example 3. Consider the system represented by digraph 3.

For this example, in the case where control inputs are considered to be generically constant, we have $\rho(\mathbf{W}, \mathbf{Y})=2$ and $\rho\left(\mathbf{W} \cup\left\{\mathbf{f}_{\mathbf{1}}\right\}, \mathbf{Y}\right)=2 \neq \rho(\mathbf{W}, \mathbf{Y})+1$. We can then conclude that the detection of fault $f_{1}$ with $u_{1}(t)=u_{1}$ (generic) is impossible. But, if we consider the system with $u_{1}(t)=0$, then we obtain $\rho(\mathbf{W}, \mathbf{Y})=1$ and $\rho\left(\mathbf{W} \cup\left\{\mathbf{f}_{\mathbf{1}}\right\}, \mathbf{Y}\right)=2=\rho(\mathbf{W}, \mathbf{Y})+1$. Condition of proposition 1 is satisfied and so it is possible to detect fault $f_{1}$. The next part is dedicated to the formulation of this particular case in general contest.

\subsection{Case of some cancelled inputs}

Our objective hereafter is to characterize which control inputs have to be set to zero or not in order to detect $f_{1}$. At this aim let us denote by $T=\left\{i \mid u_{i} \neq 0\right\}$ the set of control inputs not fixed to zero. In this case, structured linear system $\Sigma_{\Lambda}^{1}$ is equivalent to a second structured linear system $\Sigma_{\Lambda}^{1}(T)$ given by :

$$
\Sigma_{\Lambda}^{1}(T):\left\{\begin{array}{l}
\dot{x}(t)=\hat{A}_{0}(T) x(t)+E f_{1}(t)+H w(t) \\
y(t)=C x(t)
\end{array}\right.
$$

where $\hat{A}_{0}(T)=A_{0}+\sum_{i \in T} u_{i} A_{i}$.

The digraph associated to system $\Sigma_{\Lambda}^{1}(T)$ is now defined by $\mathcal{G}\left(\Sigma_{\Lambda}^{1}(T)\right)=(\mathcal{V}, \mathcal{E}(T))$ with $\mathcal{E}(T)=$ $\bar{A}_{0}$-edges $\bigcup_{i \in T} \bar{A}_{i}$-edges.

We denote by $\rho_{u}\left(\mathbf{V}_{\mathbf{0}}, \mathbf{V}_{\mathbf{1}}, T\right)$ the maximum number of disjoint paths from $\mathbf{V}_{\mathbf{0}}$ to $\mathbf{V}_{\mathbf{1}}$ assuming all the control inputs generically constant except for $u_{j}$ with $j \notin T$ such that $u_{j}=0$. From the results of (Commault et al., 2002), the following proposition is given.

\section{Proposition 2 :}

Consider SBLs $\Sigma_{\Lambda}$. In the case where all the control input components are constant, SBLS $\Sigma_{\Lambda}$ is equivalent to structured linear system $\Sigma_{\Lambda}^{1}$. The active-BFPRG is then solvable if :

$$
\exists T \text { such that } \rho_{u}\left(\mathbf{W} \cup\left\{\mathbf{f}_{\mathbf{1}}\right\}, \mathbf{Y}, T\right)>\rho_{u}(\mathbf{W}, \mathbf{Y}, T)
$$

Since proposition 2 can be generally verified for different subsets $T$, it is interesting to set a procedure in 
order to obtain all subsets $T$ which enable the fault detection.

\subsection{Characterization of all solutions}

If we consider that $\rho\left(\mathbf{W} \cup\left\{\mathbf{f}_{\mathbf{1}}\right\}, \mathbf{Y}\right)=\rho(\mathbf{W}, \mathbf{Y})$, we can deduce that linkings belonging to $\mathcal{M}(\mathbf{W}, \mathbf{Y})$ of cardinality $l_{1}$ belong necessarily to $\mathcal{M}\left(\mathbf{W} \cup\left\{\mathbf{f}_{\mathbf{1}}\right\}, \mathbf{Y}\right)$ of cardinality $l_{2}$ then $\mathcal{M}(\mathbf{W}, \mathbf{Y}) \subseteq \mathcal{M}\left(\mathbf{W} \cup\left\{\mathbf{f}_{\mathbf{1}}\right\}, \mathbf{Y}\right)$ and $l_{2} \geq l_{1}$. Let us assume that $\mathcal{M}(\mathbf{W}, \mathbf{Y})=\mathrm{m}_{1} \cup \ldots \cup \mathrm{m}_{l_{1}}$ and $\mathcal{M}\left(\mathbf{W} \cup\left\{\mathbf{f}_{\mathbf{1}}\right\}, \mathbf{Y}\right)=\mathfrak{m}_{1} \cup \ldots \cup \mathfrak{m}_{l_{1}} \cup \ldots \cup \mathfrak{m}_{l_{2}}$. Linkings $\mathrm{m}_{i}$ for $i=1, \ldots, l_{1}$ do not contain any path from $\mathbf{f}_{\mathbf{1}}$ to Y. By the way, linkings $\mathrm{m}_{i}$ for $i=l_{1}+1, \ldots, l_{2}$ contain necessarily a path from $\mathbf{f}_{\mathbf{1}}$ to $\mathbf{Y}$.

If $l_{2}=l_{1}$, we can conclude that there is no path from $\mathbf{f}_{\mathbf{1}}$ to $\mathbf{Y}$ and consequently, fault $f_{1}$ cannot be detected without adding a new sensor.

Considering now a maximum linking containing a path from $\mathbf{f}_{\mathbf{1}}$, the proposed approach consists in comparing this linking with all other maximum linkings (with no path from $\mathbf{f}_{\mathbf{1}}$ ). This comparison needs some new definitions.

- Three operations $\odot, \oplus$ and $\oslash$ on set $U=\left\{u_{0}, \ldots, u_{\mathbf{m}}\right\}$ are defined. $\odot$ and $\oplus$ are commutative and associative.

- To path $P=\mathbf{v}_{\mathbf{r}_{0}} \stackrel{u_{i_{1}}}{\longrightarrow} \mathbf{v}_{\mathbf{r}_{1}} \ldots \mathbf{v}_{\mathbf{r}_{\mathbf{k}-1}} \stackrel{u_{i k}}{\longrightarrow} \mathbf{v}_{\mathbf{r}_{\mathbf{k}}}$, we associate the expression $\psi(P)=\mathbf{u}_{\mathbf{i}_{1}} \odot \mathrm{u}_{\mathbf{i}_{2}} \odot \ldots \odot \mathbf{u}_{\mathbf{i}_{\mathbf{k}}}$.

- Considering $\mathrm{u}^{\mathbf{1}}=\mathrm{u}$, the following relations are used in order to simplify expressions.

$$
\begin{aligned}
& u^{\mathbf{k}_{1}} \odot \mathbf{u}^{\mathbf{k}_{2}}=\mathbf{u}^{\mathbf{k}_{1}+\mathbf{k}_{2}} \text { with } k_{1} \geq 1 \text { and } k_{2} \geq 1 . \\
& \left(u_{\mathbf{i}_{1}}^{\mathbf{k}_{1}} \odot \psi_{1}\right) \oslash\left(u_{\mathbf{i}_{1}}^{\mathbf{k}_{3}} \odot \psi_{2}\right)=\left\{\begin{array}{l}
\left(\mathbf{u}_{\mathbf{i}_{1}} \mathbf{k}_{\mathbf{1}} \odot \psi_{1}\right) \oslash \psi_{2} \text { if } k_{1}>k_{3} . \\
\psi_{1} \oslash\left(\mathbf{u}_{\mathbf{i}_{1}}^{\mathbf{k}_{3}-\mathbf{k}_{1}} \odot \psi_{2}\right) \text { if } k_{1}<k_{3} . \\
\psi_{1} \oslash \psi_{2} \text { if } k_{1}=k_{3} .
\end{array}\right.
\end{aligned}
$$

- The complement operation on expression $\psi$ is denoted by $\bar{\psi}$ with the following properties : $\overline{u_{1} \odot u_{2} \odot \ldots \odot u_{k}}=\bar{u}_{1} \oplus \bar{u}_{2} \oplus \ldots \oplus \bar{u}_{k}$ and $\overline{u_{1} \oplus u_{2} \oplus \ldots \oplus u_{k}}=\bar{u}_{1} \odot \bar{u}_{2} \odot \ldots \odot \bar{u}_{k}$.

- To each maximum linking $m_{i} \in \mathcal{M}\left(\mathbf{V}_{\mathbf{0}}, \mathbf{V}_{\mathbf{1}}\right)$, we associate the expression $\psi\left(\mathrm{m}_{i}\right)=\bigodot_{P_{j} \in \mathfrak{m}_{i}} \psi\left(P_{j}\right)$. The latter enables to know which control inputs appear in the maximum linking $m_{i}$. The interest is that we can deduce which control inputs could be set to zero in order to decrease the size of $\mathrm{m}_{i}$.

- Function $\operatorname{cp}\left(\mathfrak{m}_{i}, \mathfrak{m}_{\ell}\right)$ makes the comparison between linkings $m_{i}$ and $\mathfrak{m}_{\ell}$. It is defined such that:

If $\psi_{1} \oslash \psi_{2}$ is the expression of $\psi\left(m_{i}\right) \oslash \psi\left(m_{\ell}\right)$ after all simplifications then $\operatorname{cp}\left(\mathfrak{m}_{i}, \mathfrak{m}_{\ell}\right)=\psi_{1}$.

- $P_{f}(\ell)$ denotes the path from $\mathbf{f}_{\mathbf{1}}$ belonging to $\mathrm{m}_{\ell}$. $\psi\left(P_{f}(\ell)\right)$ enables to know which control inputs appear in the path from $\mathbf{f}_{\mathbf{1}}$ to $\mathbf{Y}$. The interest is that we can deduce which control inputs could be different from zero in order to keep path $P_{f}(\ell)$ in $m_{\ell}$.

More precisely, we will be able to find which control inputs have to be equal to zero and which control inputs have to be different from zero in order to obtain all the possible solutions by computing the set $\mathcal{S}$ as below.

$N(\ell)$ indicates the control inputs belonging to $\psi\left(\mathrm{m}_{i}\right)$ for $i=1, \ldots, l_{1}$ and not to $\psi\left(\mathrm{m}_{\ell}\right)$. The complement of $N(\ell)$ correspond to the control inputs which can be set to zero without deleting the link between $\mathbf{f}_{\mathbf{1}}$ and $\mathbf{Y}$.

Furthermore, $D(\ell)$ indicates which control inputs have to be different from zero in order to keep the path from $\mathbf{f}_{\mathbf{1}}$ in the linking $\boldsymbol{m}_{\ell}$.

From a computational point of view, $N(\ell)$ and $D(\ell)$ can be determined by mean of the following algorithm.

For $\ell=l_{1}+1$ to $l_{2}$
$N(\ell)=\bigoplus_{i=1}^{l_{1}} \mathrm{cp}\left(\psi\left(\mathrm{m}_{i}\right), \psi\left(\mathrm{m}_{\ell}\right)\right)$
$D(\ell)=\psi\left(P_{f}(\ell)\right)$

where maximum linkings $m_{i}$ do not contain any path from $\mathbf{f}_{\mathbf{1}}$ and maximum linkings $\boldsymbol{m}_{\ell}$ contain a path from $\mathbf{f}_{1}$.

According to the general form of $\bar{N}(\ell)$ which is $\left(\bar{u}_{\mathbf{k}_{1}}^{\mathbf{l}_{1}} \odot\right.$ $\left.\bar{u}_{\mathbf{k}_{\mathbf{2}}}^{\mathbf{l}_{\mathbf{2}}} \odot \ldots \odot \bar{u}_{\mathbf{k}_{\mathbf{s}}}^{\mathbf{l}_{\mathbf{s}}}\right) \oplus\left(\bar{u}_{\mathbf{k}_{11}}^{\mathbf{l}_{11}} \odot \ldots \odot \bar{u}_{\mathbf{k}_{\mathbf{t}}}^{\mathbf{l}_{\mathbf{t}}}\right) \oplus \ldots$, we create the set $\mathcal{S}(\ell)=\left\{\left\{\bar{u}_{\mathbf{k}_{1}}, \bar{u}_{\mathbf{k}_{2}}, \ldots, \bar{u}_{\mathbf{k}_{\mathrm{s}}}\right\},\left\{\bar{u}_{\mathbf{k}_{11}}, \bar{u}_{\mathbf{k}_{12}}, \ldots\right\}, \ldots\right\}$.

Finally, if $D(\ell)$ is on the form $u_{\mathbf{c}_{1}}^{\mathbf{d}_{1}} \odot \mathrm{u}_{\mathbf{c}_{2}}^{\mathbf{d}_{2}} \odot \ldots \odot$ $\mathrm{u}_{\mathbf{c}_{\mathrm{v}}}^{\mathbf{d}_{\mathbf{v}}}$, we modify set $\mathcal{S}(\ell)$ by adding $\mathrm{u}_{\mathbf{c}_{1}}, \mathrm{u}_{\mathbf{c}_{2}}, \ldots, \mathrm{u}_{\mathbf{c}_{\mathrm{v}}}$ to all elements of $\mathcal{S}(\ell)$ in order to obtain $\mathcal{S}(\ell)=$ $\left\{\left\{\bar{u}_{\mathbf{k}_{1}}, \bar{u}_{\mathbf{k}_{2}}, \ldots, \bar{u}_{\mathbf{k}_{\mathrm{s}}}, \mathrm{u}_{\mathbf{c}_{1}}, \mathrm{u}_{\mathbf{c}_{2}}, \ldots, \mathrm{u}_{\mathbf{c}_{\mathrm{v}}}\right\}, \ldots\right\}$.

Each element of $\mathcal{S}(\ell)$ is composed of elements $\bar{u}_{\mathbf{d}}$ and $u_{\mathbf{e}}$. Elements $\bar{u}_{\mathbf{d}}$ give control inputs having to be set to zero and $u_{\mathrm{e}}$ gives control inputs having to be different from zero.

From a practical point of view, it is important to remove some elements of $\mathcal{S}(\ell)$ according to the following rules :

- if an element $s_{g}$ of $\mathcal{S}(\ell)$ contains $\bar{u}_{0}$ then $\mathcal{S}(\ell)=$ $\mathcal{S}(\ell) \backslash\left\{s_{g}\right\}$, since $\mathrm{u}_{0}$ is generically different from zero because it depends on the system parameters.

- if an element $s_{h}$ of $\mathcal{S}(\ell)$ contains $\bar{u}_{\mathbf{i}}$ and $u_{\mathbf{i}}$ then $\mathcal{S}(\ell)=\mathcal{S}(\ell) \backslash\left\{s_{h}\right\}$, since control inputs cannot be set to zero and different from zero at the same time.

\section{Proposition 3}

Consider sBLs $\Sigma_{\Lambda}$. In the case where all the control input components are constant, SBLs $\Sigma_{\Lambda}$ is equivalent to structured linear system $\Sigma_{\Lambda}^{1}$. The active-BFPRG is then solvable if :

$$
\mathcal{S}=\bigcup_{\ell=l_{1}+1}^{l_{2}} \mathcal{S}(\ell) \neq \emptyset
$$

Moreover, all the solutions are given by set $\mathcal{S}$. Let $s_{1}=\left\{\bar{u}_{\mathbf{i}}, \ldots, u_{\mathbf{j}}, \ldots\right\}$ be an element of $\mathcal{S}$. If $\bar{u}_{\mathbf{i}} \in s_{1}$ then $u_{i}$ have to be set to zero. If $u_{\mathbf{j}} \in s_{1}$ then $u_{j}$ have to be different from zero. If $u_{\mathbf{k}} \notin s_{1}$ and $\bar{u}_{\mathbf{k}} \notin s_{1}$ then $u_{k}$ can be indifferently set to zero or different from zero. 


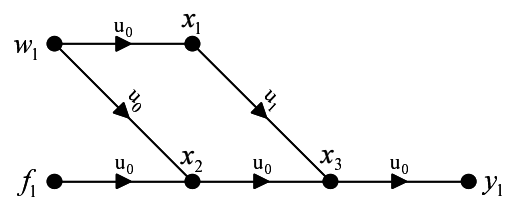

Figure 4. Particular digraph for BFPRG solvability

Example 3.

In example 3, there are 4 paths from $\mathbf{W} \cup\left\{\mathbf{f}_{\mathbf{1}}\right\}$ to $\mathbf{Y}$. $P_{1}=\mathbf{w}_{\mathbf{1}} \stackrel{u_{0}}{\longrightarrow} \mathbf{x}_{\mathbf{1}} \stackrel{u_{2}}{\longrightarrow} \mathbf{x}_{\mathbf{4}} \stackrel{u_{0}}{\longrightarrow} \mathbf{y}_{\mathbf{1}}, \psi\left(P_{1}\right)=\mathrm{u}_{\mathbf{0}}^{2} \odot \mathrm{u}_{\mathbf{2}}$. $P_{2}=\mathbf{w}_{\mathbf{2}} \stackrel{u_{0}}{\longrightarrow} \mathbf{x}_{\mathbf{2}} \stackrel{u_{1}}{\longrightarrow} \mathbf{x}_{\mathbf{5}} \stackrel{u_{0}}{\longrightarrow} \mathbf{y}_{\mathbf{2}}, \psi\left(P_{2}\right)=\mathrm{u}_{\mathbf{0}}^{\mathbf{2}} \odot \mathrm{u}_{\mathbf{1}}$.

$P_{3}=\mathbf{w}_{2} \stackrel{u_{0}}{\longrightarrow} \mathbf{x}_{2} \stackrel{u_{0}}{\longrightarrow} \mathbf{x}_{\mathbf{4}} \stackrel{u_{0}}{\longrightarrow} \mathbf{y}_{\mathbf{1}}, \psi\left(P_{3}\right)=\mathbf{u}_{\mathbf{0}}^{\mathbf{3}}$.

$P_{4}=\mathbf{f}_{1} \stackrel{u_{0}}{\longrightarrow} \mathbf{x}_{\mathbf{3}} \stackrel{u_{2}}{\longrightarrow} \mathbf{x}_{\mathbf{5}} \stackrel{u_{0}}{\longrightarrow} \mathbf{y}_{2}, \psi\left(P_{4}\right)=\mathrm{u}_{\mathbf{0}}^{2} \odot \mathrm{u}_{\mathbf{2}}$.

Then, there are 3 maximum linkings from $\mathbf{W} \cup\left\{\mathbf{f}_{\mathbf{1}}\right\}$ to $\mathbf{Y}$ and 1 maximum linking from $\mathbf{W}$ to $\mathbf{Y}$.

$\mathcal{M}\left(\mathbf{W} \cup\left\{\mathbf{f}_{\mathbf{1}}\right\}, \mathbf{Y}\right)=\left\{P_{1}, P_{2}\right\} \cup\left\{P_{1}, P_{4}\right\} \cup\left\{P_{3}, P_{4}\right\}=m_{1} \cup$ $\mathrm{m}_{2} \cup \mathrm{m}_{3} .\left(l_{2}=3\right)$

$\mathcal{M}(\mathbf{W}, \mathbf{Y})=\mathrm{m}_{1} \cdot\left(l_{1}=1\right)$

$\psi\left(\mathrm{m}_{1}\right)=\psi\left(P_{1}\right) \odot \psi\left(P_{2}\right)=\mathrm{u}_{0}^{4} \odot \mathrm{u}_{1} \odot \mathrm{u}_{2}$.

$\psi\left(\mathrm{m}_{2}\right)=\psi\left(P_{1}\right) \odot \psi\left(P_{4}\right)=\mathrm{u}_{\mathbf{0}}^{4} \odot \mathrm{u}_{\mathbf{2}}^{\mathbf{2}}$.

$\psi\left(\mathrm{m}_{3}\right)=\psi\left(P_{3}\right) \odot \psi\left(P_{4}\right)=\mathrm{u}_{0}^{5} \odot \mathrm{u}_{2}$.

Now, the method consists in compare $m_{2}$ and $m_{3}$ with $\mathrm{m}_{1}$ by the computation of $\mathcal{S}$.

$N(2)=\operatorname{cp}\left(\mathrm{m}_{1}, \mathrm{~m}_{2}\right)=\mathrm{u}_{\mathbf{1}}, \bar{N}(2)=\bar{u}_{1}, D(2)=\psi\left(P_{4}\right)=$ $\mathrm{u}_{0}^{2} \odot \mathrm{u}_{2}$ and then $\mathcal{S}(2)=\left\{\overline{\mathrm{u}}_{1}, \mathrm{u}_{\mathbf{0}}, \mathrm{u}_{2}\right\}$.

$N(3)=\operatorname{cp}\left(\mathrm{m}_{1}, \mathrm{~m}_{3}\right)=\mathrm{u}_{1}, \bar{N}(2)=\overline{\mathrm{u}}_{1}, D(3)=\psi\left(P_{4}\right)=$ $\mathrm{u}_{0}^{2} \odot \mathrm{u}_{2}$ and then $\mathcal{S}(3)=\left\{\overline{\mathrm{u}}_{1}, \mathrm{u}_{\mathbf{0}}, \mathrm{u}_{2}\right\}$.

$\mathcal{S}=\left\{\overline{\mathrm{u}}_{1}, \mathrm{u}_{0}, \mathrm{u}_{2}\right\}$. We conclude that the active-BFPRG is solvable if $u_{1}=0$ and $u_{2}=$ constant $\neq 0$ ( since $u_{0}$ is generically different from zero).

\subsection{Remark}

There also exists the case of particular constant inputs (but not equal to zero) making the fault detection possible. For example, from the system represented in figure 4 , we can write a relation on the form $\ddot{y}_{1}(t)=$ $\alpha_{1} f_{1}(t)+\alpha_{2} w_{1}(t)+\alpha_{3} u_{1}(t) w_{1}(t)+\alpha_{4} \dot{u}_{1}(t) x_{1}(t)$ (with $\alpha_{i}$ depending on the system parameters) and then if $u_{1}(t)$ is equal to the appropriate constant $\left(u_{1}(t)=\frac{-\alpha_{2}}{\alpha_{3}}\right)$ we obtain $\ddot{y}_{1}(t)=\alpha_{1} f_{1}(t)$.

We see that a very particular value for the control input makes the fault detectable but this particular value can only be obtained from the knowledge of the parameters.

Consequently, this case is not in adequation with the fact that we consider structured systems, which means that we do not know the parameters value.

\section{CONCLUSION}

In this paper, we propose a new analysis tool to check the solvability of the BFPRG considering the control input influence. For this purpose, sufficient conditions to the existence of input values making the so-called active-BFPR solvable have been provided on the basis of graph-theoretic approach.

All the conditions we have enounced in this paper do not depend on the parameters of the system and have an intuitive interpretation. Moreover, they are very easy to check by means of well-known combinatorial techniques and simply by hand for small systems. From a computational point of view, note that our approach is particularly suited for large-scale systems and is free from numerical difficulties. Indeed, the proposed algorithm and conditions can be easily implemented because they require simple computations on expressions and are based on finding paths in graphs. Furthermore, the graphical approach makes easy the visualization of the system structure.

In fact, we have seen that among the possibilities to make fault detectable, there are the control inputs influence and the sensor placement. In further work, we will investigate the sensor placement to fulfill the fault detection of the system when this present study do not give any solution.

\section{REFERENCES}

Chen, J. and R.J. Patton (1999). Robust model-based fault diagnosis for dynamic systems. Vol. 3 of Kluwer international series on Asian studies in computer and information science. Kluwer Academic Publishers. Boston, U.S.A.

Commault, C., J-M. Dion, O. Sename and R. Motyeian (2002). Observer-based fault diagnosis for structured systems. IEEE Transactions on Automatic Control 47(12), 2074-2079.

De Persis, C. and A. Isidori (2001). A geometric approach to nonlinear fault detection and isolation. IEEE Transactions on Automatic Control 46(6), $853-865$.

Dion, J-M., C. Commault and J. Van der Woude (2003). Generic properties and control of linear structured systems: A survey. Automatica 39(7), 1125-1144.

Frank, P.M. (1996). Analytical and qualitative modelbased fault diagnosis - a survey and some new results. European Journal of Control 2(1), 6-28.

Gertler, J. (1998). Fault detection and diagnosis in engineering systems. Marcel Dekker. New York, U.S.A.

Hammouri, H., M. Kinnaert and E. H. El Yaagoubi (1999). Observer based approach to fault detection and isolation for non linear systems. IEEE Transactions on Automatic Control 44(10), $1879-1884$.

Hammouri, H., P. Kabore and M. Kinnaert (2001). A geometric approach to fault detection and isolation for bilinear systems. IEEE Transactions on Automatic Control 46(9), 1451-1455.

Massoumnia, M. A., G. C. Verghese and A. S. Willsky (1989). Failure detection and identification. IEEE Transactions on Automatic Control 34(3), 316321. 
Yang, H. and M. Saif (1995). State observation, failure detection and isolation (FDI) in bilinear systems. In: Proceedings of the 34th IEEE Conference on Decision and Control. New Orleans, U.S.A.. pp. 2391-2396. 